\title{
Feline Coronavirus 3c Protein: A Candidate for a Virulence Marker?
}

\author{
A. S. Hora, ${ }^{1,2}$ P. O. Tonietti, ${ }^{3}$ S. A. Taniwaki, ${ }^{2}$ K. M. Asano, ${ }^{1,2}$ P. Maiorka, ${ }^{3}$ \\ L. J. Richtzenhain,, ${ }^{1,2}$ and P. E. Brandão ${ }^{1,2}$ \\ ${ }^{1}$ Department of Preventive Veterinary Medicine and Animal Health, School of Veterinary Medicine, University of São Paulo, \\ Avenida Professor Dr. Orlando Marques de Paiva 87, Cidade Universitária, 05508-270 São Paulo, SP, Brazil \\ ${ }^{2}$ Coronavirus Research Group, School of Veterinary Medicine, University of São Paulo, \\ Avenida Professor Dr. Orlando Marques de Paiva 87, Cidade Universitária, 05508-270 São Paulo, SP, Brazil \\ ${ }^{3}$ Department of Pathology, School of Veterinary Medicine, University of São Paulo, \\ Avenida Professor Dr. Orlando Marques de Paiva 87, Cidade Universitária, 05508-270 São Paulo, SP, Brazil
}

Correspondence should be addressed to A. S. Hora; alineshora@gmail.com

Received 11 February 2016; Revised 1 April 2016; Accepted 10 April 2016

Academic Editor: Nikolai V. Ravin

Copyright (c) 2016 A. S. Hora et al. This is an open access article distributed under the Creative Commons Attribution License, which permits unrestricted use, distribution, and reproduction in any medium, provided the original work is properly cited.

Feline infectious peritonitis virus (FIPV) is highly virulent and responsible for the highly fatal disease feline infectious peritonitis (FIP), whereas feline enteric coronavirus (FECV) is widespread among the feline population and typically causes asymptomatic infections. Some candidates for genetic markers capable of differentiating these two pathotypes of a unique virus (feline coronavirus) have been proposed by several studies. In the present survey, in order to search for markers that can differentiate FECV and FIPV, several clones of the $3 \mathrm{a}-\mathrm{c}, \mathrm{E}$, and $\mathrm{M}$ genes were sequenced from samples obtained from cats with or without FIP. All genes showed genetic diversity and suggested the presence of FCoV mutant spectrum capable of producing a virulent pathotype in an individualspecific way. In addition, all the feline coronavirus FIPV strains demonstrated a truncated $3 \mathrm{c}$ protein, and the $3 \mathrm{c}$ gene was the only observed pathotypic marker for FCoVs, showing that $3 \mathrm{c}$ gene is a candidate marker for the distinction between the two pathotypes when the mutant spectrum is taken into account.

\section{Introduction}

Feline coronavirus (FCoV) (Nidovirales; Coronaviridae; Coronavirinae; Alphacoronavirus 1) is a widespread pathogen among the domestic cat population. FCoV occurs as two pathotypes, feline enteric coronavirus (FECV) and feline infectious peritonitis virus (FIPV), both of which with strains of serotypes I and II [1].

Phenotypically, the main difference between the pathotypes is that FIPV is able to infect monocytes and macrophages, causing systemic infection and fatal disease, while FECV is limited to replication in the mature intestinal epithelium, which mainly results in asymptomatic infection. However, FECV may undergo a short systemic viremia phase that also involves monocytes $[2,3]$.

The FCoV genome (single-stranded positive-sense linear RNA, $29 \mathrm{~kb}$ ) encodes proteins directly involved in viral replication (ORF1), the structural spike protein (S), envelope protein $(\mathrm{E})$, membrane protein $(\mathrm{M})$, and nucleocapsid protein $(\mathrm{N})$, as well as the accessory proteins $3 \mathrm{a}-\mathrm{c}$ and $7 \mathrm{a}-\mathrm{b}[4]$.

There is a major controversy regarding the existence of genetic markers to differentiate between FECV and FIPV pathotypes $[5,6]$. Numerous markers that correlate with the manifestation of FIP have been proposed for $S$ [7-12], 3a-c [8, 13-16], 7a-b [16-18], and M [5] genes.

The most studied gene is $3 c[7,9,13-15,19,20]$, which is present in the truncated form in the majority of all strains obtained from animals with FIP. However, previous studies on $3 \mathrm{c}$ genes focused only on the dominant sequence and not on the mutant spectrum or the clonal diversity and the information obtained from a dominant sequence may not be suitable to detect mutations [10], with the exception of a study [7] in which clones were used to assess the presence of alterations in the gene $3 c$ in ascites and/or faeces from FIP and 
healthy cats, and again $3 c$ truncated protein was not observed in $100 \%$ of FIPV samples.

Considering (i) the current discussion on molecular markers capable of distinguishing the pathotypes of FCoV and (ii) the lack of knowledge of the diversity of such markers in the mutant spectrum of RNA, the present study was designed to investigate the clonal diversity of the $3 \mathrm{a}-\mathrm{c}, \mathrm{E}$, and $\mathrm{M}$ genes in FCoV strains present in tissue and/or faecal samples from cats with and without FIP.

\section{Materials and Methods}

2.1. Animals and Samples. From 2010 to 2013, tissue (eye, cerebrum, cerebellum, lung, heart muscle, thoracic lymph node, thymus, liver, spleen, stomach, mesenteric lymph node, peripancreatic lymph node, kidneys, large and small intestines, and urinary bladder), abdominal, thoracic, and pericardial effusions, aqueous humor, and faecal samples were collected during the necropsies of 6 cats with clinical manifestations and histopathological changes consistent with FIP, such as localized inflammation with macrophages, neutrophils, lymphocytes, and plasma cells. Vascular lesions were found surrounded by a proliferation of inflammatory cells in heart, lung, omentum, kidneys, cerebrum, and cerebellum. Focal accumulations of inflammatory cells and necroticproliferative lesions were observed in granulomatous lesions. Intestinal samples were collected including mucosa, submucosa, muscular layer, and serosa. Only 2 animals (USP6, and USP7) showed signs of dry-form FIP (neurological changes), while the remaining 4 (USP8, USP16, USP17, and USP18) showed an accumulation of effusion in body cavities (effusive FIP).

All FIP-positive cats were sampled from different geographic locations, with the exception of 2 cats (USP17 and USP18) that lived in the same house. USP17 was euthanized 1 month prior to USP18 due to FIP related reasons.

In parallel, faecal samples were also obtained from 2 cats (USP2, and USP14) without clinical signs of FIP and from two different locations not related to the locations from the FIP+ cats.

This study has been approved by Commission on Ethics on Animal Use from School of Veterinary Medicine, University of São Paulo (CEUA N. 2055211113).

2.2. Messenger RNA Detection for the M Gene of FCoVs. All tissues were complete homogenized after tissue maceration and before RNA extraction. Total RNA extraction and RT-PCR to detect mRNA of the M gene of FCoVs were performed in all samples as previously described [21] as a screening test for FCoV replication.

2.3. Amplification of the 3a-c, E, and M Genes of FCoVs. All samples from 6FIP+ and 2FIP- cats were submitted to amplification of the $3 \mathrm{a}-\mathrm{c}, \mathrm{E}$, and $\mathrm{M}$ genes of FCoVs, even if the mRNA detection was negative. To amplify all these five genes of FCoVs in only one amplicon, a single pair of previously described primers [14] was used (nucleotides
TABle 1: Primers used to obtain full coverage of genes $3 a-c, E$, and M.

\begin{tabular}{lcc}
\hline Primers & Sequence $\left(5^{\prime}-3^{\prime}\right)$ & Position $^{\mathrm{a}}$ \\
\hline 2B & YATYACWGTKTAYRABTT & $487-505$ \\
3B & ACRACWYTNAKKTTTGTA & $1058-1075$ \\
4B & AATAGCATTGCTAAATRT & $1479-1496$ \\
5B & CGGCTTTAGTRTYGCAGG & $1999-2008$ \\
\hline
\end{tabular}

${ }^{a}$ Position defined according to genome FCoV-WSU 79-1146 (DQ010921).

24,380 to 26,996 corresponding to the FCoV-WSU 79-1146; genome GenBank DQ010921).

cDNA synthesis was performed with $6 \mu \mathrm{L}$ total RNA extracted with TRIzol ${ }^{\mathrm{Ts}}$ Reagent (Life Technologies Corporation, Carlsbad, CA, USA) and ThermoScript ${ }^{\mathrm{Ts}}$ (Life Technologies Corporation, Carlsbad, CA, USA) using Random Primers $^{\text {TM }}$ (Life Technologies Corporation, Carlsbad, CA, USA) according to the manufacturer's instructions.

For PCR, $5 \mu \mathrm{L}$ of cDNA was added to $1 \mathrm{x}$ PCR buffer (Life Technologies Corporation, Carlsbad, CA, USA), 2 mM $\mathrm{MgSO}_{4}, 0.2 \mathrm{mM}$ of each dNTP, $0.25 \mu \mathrm{M}$ of each primer, $1.5 \mathrm{U}$ Platinum $^{\mathrm{Ts}}$ Taq DNA Polymerase High Fidelity (Life Technologies Corporation, Carlsbad, CA, USA), and DEPC water for a final reaction volume of $50 \mu \mathrm{L}$. The cycling conditions were as follows: $94^{\circ} \mathrm{C} / 2 \mathrm{~min} ; 10$ cycles of $94^{\circ} \mathrm{C} / 15 \mathrm{~s}, 50^{\circ} \mathrm{C} / 15 \mathrm{~s}$ with an increase of $0.5^{\circ} \mathrm{C}$ per cycle, and $68^{\circ} \mathrm{C} / 4 \mathrm{~min} ; 10$ cycles of $94^{\circ} \mathrm{C} / 15 \mathrm{~s}, 55^{\circ} \mathrm{C} / 15 \mathrm{~s}$, and $68^{\circ} \mathrm{C} / 4 \mathrm{~min} ; 25$ additional cycles of $94^{\circ} \mathrm{C} / 15 \mathrm{~s}, 55^{\circ} \mathrm{C} / 15 \mathrm{~s}$, and $68^{\circ} \mathrm{C} / 4 \mathrm{~min}$ with an increase of $10 \mathrm{~s}$ per cycle; and a final extension of $72^{\circ} \mathrm{C} / 10 \mathrm{~min}$.

Specific bands were excised from the gels and purified using the Gene Jet ${ }^{\mathrm{Tm}}$ Gel Extraction kit (Thermo Fisher Scientific Inc., Vilnius, Lithuania) according to the manufacturer's instructions.

2.4. Molecular Cloning. The purified amplicons $(2,617 \mathrm{bp}$ containing 3abc-E-M) were cloned using pGEM ${ }^{\mathrm{m}}-\mathrm{T}$ Easy Vector Systems (Promega Madison, WI, USA) and E. coli JM109 competent cells according to the manufacturer's recommendations. After the transformation protocol, transformation culture was plated onto duplicate LB/ampicillin/ IPTG/X-Gal plates and the plates were incubated overnight (16 hours) at $37^{\circ} \mathrm{C}$. Then, the transformed colonies were transferred to LB liquid medium and were incubated overnight (16 hours) at $37^{\circ} \mathrm{C}$ and $180 \mathrm{rpm}$. Recombinant plasmid extraction was performed with the Illustra ${ }^{\mathrm{Tm}}$ plasmidPrep Mini Spin Kit (GE Healthcare, Buckinghamshire, UK) as per manufacturer's recommendations.

For an easier identification of the clones, the following nomenclature was used: cat identification/sample source/ presence $(+)$ or absence $(-)$ of FIP/cat origin/clone number.

2.5. DNA Sequencing. Sense primers were designed at approximately every 500 nucleotides to obtain full coverage of genes $3 \mathrm{a}-\mathrm{c}, \mathrm{E}$, and $\mathrm{M}$ (Table 1). Primers for the plasmid ( $\mathrm{pUC} / \mathrm{M} 13 \mathrm{~F}$ and $\mathrm{R}$ ) were also used to reach the full length of the amplicon sequence. 
TABLE 2: Identification, sorting according to the presence of FIP detection of mRNA FCoVs, and the number of clones obtained from cats.

\begin{tabular}{|c|c|c|c|c|c|c|c|c|c|c|}
\hline \multirow{3}{*}{ ID } & \multirow{3}{*}{ Location } & \multirow{3}{*}{ FIP } & \multirow{3}{*}{ Sample } & \multirow{3}{*}{ mRNA } & \multicolumn{5}{|c|}{ Number of clones obtained from each gene } & \multirow{3}{*}{ Total } \\
\hline & & & & & $\mathrm{E}$ & M & $3 a$ & $3 b$ & $3 c$ & \\
\hline & & & & & 249nt & $792 n t$ & $213 n t$ & $222 n t$ & $714 n t$ & \\
\hline USP2 & 1 & Absent & Faeces & Pos & 4 & 6 & 6 & 6 & 4 & 26 \\
\hline \multirow{2}{*}{ USP6 } & \multirow{2}{*}{7} & \multirow{2}{*}{ Noneffusive } & Stomach & Pos & 10 & 11 & 10 & 9 & 6 & 46 \\
\hline & & & Faeces & Neg & 0 & 3 & 8 & 7 & 5 & 23 \\
\hline \multirow{2}{*}{ USP7 } & \multirow{2}{*}{3} & \multirow{2}{*}{ Noneffusive } & Cerebrum & $\mathrm{Neg}$ & 5 & 5 & 5 & 5 & 2 & 22 \\
\hline & & & Faeces & Pos & 3 & 7 & 10 & 10 & 5 & 35 \\
\hline \multirow{2}{*}{ USP8 } & \multirow{2}{*}{6} & \multirow{2}{*}{ Effusive } & Stomach & Pos & 1 & 1 & 1 & 1 & 1 & 5 \\
\hline & & & Urinary bladder & Pos & 0 & 0 & 3 & 3 & 2 & 8 \\
\hline \multirow{2}{*}{ USP17 } & \multirow{2}{*}{2} & \multirow{2}{*}{ Effusive } & Cerebrum & Neg & 3 & 5 & 8 & 8 & 8 & 32 \\
\hline & & & Large intestine & Pos & 3 & 1 & 4 & 4 & 2 & 14 \\
\hline USP14 & 4 & Absent & Faeces & Pos & 1 & 1 & 12 & 12 & 9 & 35 \\
\hline USP16 & 5 & Effusive & Cerebrum & Pos & 6 & 0 & 9 & 9 & 9 & 33 \\
\hline USP18 & 2 & Effusive & Cerebrum & Pos & 2 & 3 & 3 & 3 & 2 & 13 \\
\hline \multicolumn{3}{|c|}{ Total of clones $(n=292)$} & & & 38 & 43 & 79 & 77 & 55 & 292 \\
\hline
\end{tabular}

ID: identification; Pos: positive; Neg: negative.

DNA sequencing with each primer in duplicate was performed using the BigDye ${ }^{\mathrm{TM}}$ Terminator v3.1 Cycle Sequencing Kit (Life Technologies Corporation, Carlsbad, CA, USA) with a Genetic Analyzer $3500^{\mathrm{Tm}}$ capillary sequencer (Applied Biosystems Foster City, CA, USA), according to the manufacturers' instructions.

2.6. Sequence Editing. Chromatograms were generated in duplicate for each segment and evaluated for their base quality using the online application Phred (http://asparagin .cenargen.embrapa.br/phph/). Only those chromatograms with scores greater than 20 (probability lower than 1 error per 100 nucleotides) were used. The chromatograms were analyzed and manually edited with the software FinchTV version 1.4 (http://www.geospiza.com/Products/finchtv.shtml). The final sequences of each sample were obtained using the CAP contig application with the software BioEdit version 7.0.9.0 [22].

2.7. Phylogenetic Analyses. The nucleotides and amino acids corresponding to the $3 \mathrm{a}-\mathrm{c}, \mathrm{E}$, and $\mathrm{M}$ genes were aligned using the application Clustal/W in BioEdit 7.0.9.0 [22]. Clones were classified in serotypes based on comparative analysis of the amino acid sequences encoded by the $3 a$ gene with the sequences available in GenBank (serotype I: DQ160294 and DQ848678 and serotype II: DQ010921). Nucleotide phylogenetic trees (neighbor-joining, MCL model, 1,000 bootstrap replicates) were constructed using the computer software Mega version 5.05 [23].

\section{Results}

3.1. Messenger RNA Detection for the M Gene and Amplification, Sequencing, and Cloning of the $3 a-c, E$, and $M$ Genes of FCoVs. mRNA was not obtained from all samples; nevertheless sequences for the $3 \mathrm{a}-\mathrm{c}, \mathrm{E}$, and $\mathrm{M}$ amplicons were obtained from a total of 292 clones originating from 8 animals [6FIP+ (USP6, USP7, USP8, USP17, USP16, and USP18) and 2FIP- (USP2 and USP14)]. Sequences from extraintestinal tissue samples and faecal/intestines samples were obtained simultaneously for 3 animals (USP6, USP7, and USP17) with FIP (Table 2). Due to poor sequence quality for some clones and/or regions in a clone, not all full length amplicons could be sequenced or used in the analyses. The sequences obtained in the present study were deposited in GenBank under access numbers KJ879334 to KJ879438.

3.2. Phylogenetic Analysis of the $3 a-c, E$, and $M$ Genes of FCoVs. All sequences of the 3 a genes obtained from a total of 79 clones belong to serotype I. Phylogenetic trees generated for the $3 \mathrm{a}, 3 \mathrm{c}, \mathrm{E}$, and $\mathrm{M}$ genes showed similar topologies and thus only the $3 \mathrm{c}$ and $3 \mathrm{~b}$ trees are shown (Figures 1 and 2, resp.). Sample from cat USP14 (non-FIP) showed a remarkable clonal diversity as their clones are present in five different clusters of the $3 \mathrm{~b}$ tree. High clonal diversity was observed in samples from FIP+ (USP8, USP16, USP17, and USP18) and FIP- cat (USP14); however FIP+ cats USP6 and USP7 and USP2 FIP- cat showed a lower clonal diversity. In the $3 c$ gene tree, for cats USP6 and USP7, differences can be noticed between the FCoV strains obtained from tissue and faecal clones; that is, for each animal, there was a distinction between systemic (FIPV) and enteric (FECV) strains. However, no generic grouping per pathotype (FECV or FIPV) was observed for all samples and trees generated from this study.

Conversely, in the $3 \mathrm{~b}$ gene tree samples obtained from cat USP7 (FIP+) could not be separated into systemic (cerebrum) and enteric (faeces) strains.

The overall nucleotide identity of all clones (FIP+ and FIP-) obtained from the 5 genes was $\geq 92.09 \%$ for faecal 


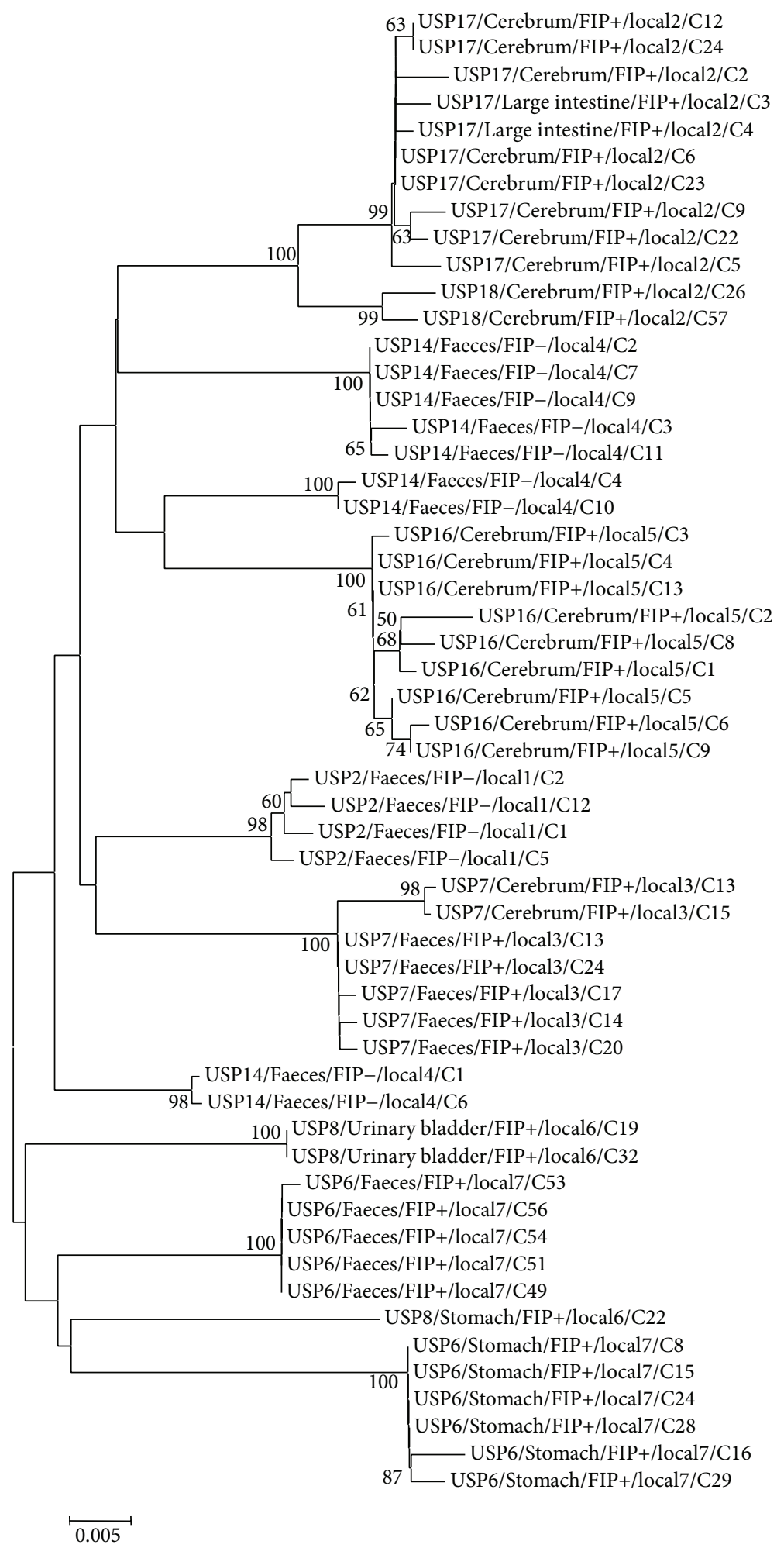

Figure 1: Phylogenetic tree constructed using the neighbor-joining method and the maximum composite likelihood substitution model for the FCoV $3 \mathrm{c}$ gene. The numbers next to each node represent the values of 1,000 bootstrap repetitions, and only those above $50 \%$ are shown. The scale represents the number of substitutions per site. For easy identification of the samples, the following nomenclature was used: cat identification/sample source/presence $(+)$ or absence (-) of FIP/cat origin/clone number. 


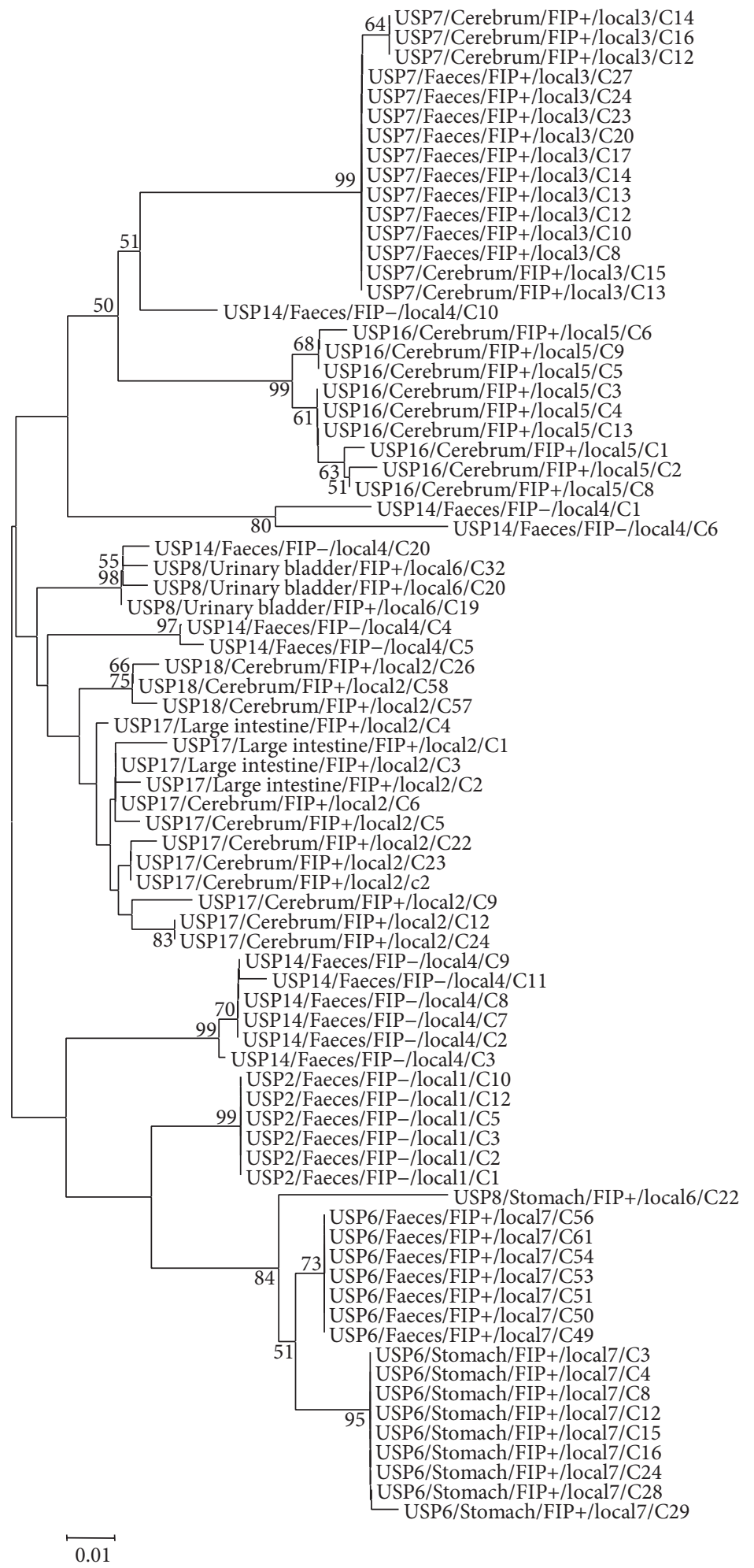

FIGURE 2: Phylogenetic tree constructed using the neighbor-joining method and the maximum composite likelihood substitution model for the FCoV $3 \mathrm{~b}$ gene. The numbers next to each node represent the values of 1,000 bootstrap repetitions, and only those above $50 \%$ are shown. The scale represents the number of substitutions per site. For easy identification of the samples, the following nomenclature was used: cat identification/sample source/presence (+) or absence (-) of FIP/cat origin/clone number. 
samples and $\geq 90.09 \%$ for tissue samples (Table 3 ). The greatest nucleotide diversity when all available sequences for each gene were compared together was observed for the 3 b gene, for which a $90.09 \%$ identity was found, with the exception of comparisons between cats living in the same house, for which the identity between the $3 \mathrm{~b}$ gene sequences was $97.09 \%$.

For tissue and faecal samples, $3 \mathrm{a}$ was the most conserved gene (nucleotide identities of $99.38 \%$ and $97.70 \%$, resp.) considering each individual cat. The mean identity between clones from the two cohabitating FIP+ cats (USP17 and USP18) ranged from $96.17 \%$ (3c gene) to $99.83 \%$ (E gene).

Premature stop codons were observed in the 3a (USP18, $3 / 3$ cerebrum clones), $3 \mathrm{~b}$ (USP17, $1 / 8$ cerebrum; USP7, $1 / 10$ faeces), $3 c$ (USP17, 8/8 cerebrum and 2/2 large intestine; USP18, 2/2 cerebrum; USP7, 2/2 cerebrum; USP16, 1/9 cerebrum; USP8, 1/1 stomach; USP6, 6/6 stomach), and M (USP7, 1/5 cerebrum) genes from FIP+ cats. No premature stop codon in the $3 \mathrm{a}-\mathrm{c}$ or E genes was observed in faecal samples from cats without FIP, with exception of M gene (USP2, 1/6 faeces).

Stop codons in $3 \mathrm{c}$ were evident in at least one of the clones from tissue samples from cats with FIP. The faecal samples from cats with or without FIP did not demonstrate this truncated gene in any of the clones, and no other molecular marker capable of differentiating the biotypes (FECV and FIPV) was observed in the sequenced genes.

\section{Discussion and Conclusions}

The existence of genetic markers to differentiate these two biotypes is currently a topic of the highest interest on the FIP field not only from the clinical and epidemiological points of view but also as a basis for an in-deep comprehension of the pathogenesis of this highly complex disease. Considering that, in the present study, clones of structural (E and $M$ ) and accessory $(3 a-c)$ genes were obtained from cats with and without FIP, and the results demonstrate a highly likely candidate $\mathrm{FCoV}$ pathotypic marker in the $3 \mathrm{c}$ gene.

Amplicons obtained from the cerebrum and large intestine of the cat USP17 (FIP+) were also cloned and sequenced. When assessing the 46 clones ( 32 clones from cerebrum and 14 from large intestine) obtained from this cat, the nucleotide identity among the five genes was greater than $99 \%$, and according to the phylogenetic trees generated, clones from this cat resulted in a single cluster. In the $3 c$ gene, a single nucleotide transversion $(\mathrm{G} \rightarrow \mathrm{T}$ ) was observed at position 10 in all clones from samples from this cat, resulting in premature stop codons and truncated proteins. The observation of truncated proteins in large intestine observed herein is in contrast to what has been observed for FECV in other studies $[13,14,20]$.

Possibly because it serves as a preferential organ for viral replication and therefore for pathological changes in FIP cases, the intestine of the cat USP17 (FIP+) was also affected by the systemic strain. Chang et al. [20] suggested that viruses with an inactivated $3 c$ gene rarely replicate in the intestine, which helps to explain the rare incidence of FIP outbreaks due to a less frequent faecal-oral transmission. A premature stop codon in the $3 \mathrm{c}$ gene was previously reported in a strain of FCoV from the jejunum of a cat with FIP, and the same intestinal strain showed $100 \%$ identity with a strain obtained from a liver sample of the same cat [6].

In recent studies $[13,15], 3 c$ genes with premature termination were detected in faecal and extraintestinal samples from cats with FIP, although it was not determined whether the viruses were replicating. Also, after an experimental inoculation of cats with truncated and complete $3 \mathrm{abc}$, the role of the truncation in this region on FIP pathogenesis could not be clearly determined, despite it led to lower viral replication in the gut [24]. However, in the present study, the sample from the large intestine of the cat USP17 (FIP+), from which the studied clones originated and which demonstrated a truncated $3 \mathrm{c}$ gene, was positive for FCoV mRNA. This result indicated the presence of viral replication in that sample.

When comparing the sequences obtained from the extraintestinal organs and faeces of the FIP+ cats USP7 and USP6, the presence of a premature stop codon in the $3 \mathrm{c}$ gene was not observed in faecal clones from both cats, in contrast to what was observed for tissue clones. Moreover, the nucleotide identity of the $3 \mathrm{c}$ gene for tissue and faecal samples was $99.09 \%$ and $95.58 \%$ for the cats USP7 and USP6, respectively. In a study comparing FCoV from extraintestinal FIP lesions and the faeces of the same cat, the identity between the sequences of the structural and accessory genes evaluated (S, M, N, E, 3a-c and 7a-b) was always higher than 99\%, corroborating the hypothesis of in vivo mutation [14].

As FIPV lineages may be derived from FECV mutations, it was expected that nucleotide diversity between faecal and tissue samples from the cat USP6 (FIP+) would be lower. However, the difference was $8.84 \%$ for the $3 \mathrm{~b}$ genes, consisting exclusively in point mutations, indicating that this cat could be coinfected with an FCoV strain that is distinct from the systemic strain. Furthermore, the high nucleotide identity between samples from the cerebrum and faeces of the cat USP7 (FIP+), along with the topology of the generated trees, showed that the intestinal viral replication of the enteric pathotype may be maintained even during FIP in some cases, as demonstrated by the presence of mRNA.

As previously described and according with results from the present study, FIPV can both emerge endogenously and be transmitted to different cats as a "ready" virulent pathotype, which has major implications for the understanding of the dynamics of viral transmission $[5,15,21]$.

Among the structural proteins genes ( $\mathrm{E}$ and $\mathrm{M})$ studied herein, premature stop codons were detected only in the $\mathrm{M}$ gene. The M protein of FCoVs is the most abundant structural protein [25]. The presence of a premature stop codon in a constitutive gene such as the $\mathrm{M}$ gene is incompatible with the functionality of the virion. However, this defective virus is no less important than the others, as it provides genetic background for recombination with other FCoVs, resulting in greater genetic diversity and possibly increased virulence of some viral quasispecies. Recent studies suggest that the $3 c$ gene, which encodes a small protein (238aa) of unknown function, is associated with most FIPV isolates when present in truncated form, although not all FCoV strains in FIP+ samples present this $3 \mathrm{c}$ form $[13,14,20]$. In the present study, the $3 \mathrm{c}$ truncated protein was observed in all clones obtained 


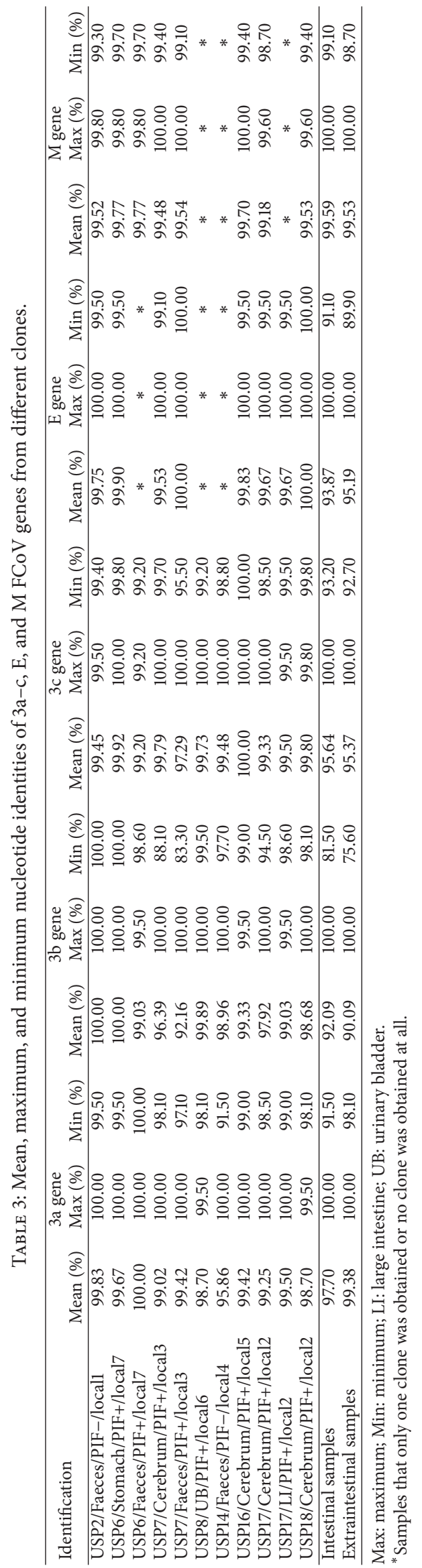


from tissue samples from FIP+ cats, with the exception of those obtained from the cat USP16, where only $11 \%$ (1/9) of the clones demonstrated the truncated protein.

It is essential to note that if the predominant viral population in the present study had been assessed by direct amplicon sequencing only, as in studies conducted by other authors $[13-15,19,20]$, without using the previous cloning step, the truncated $3 \mathrm{c}$ gene could have been undetected as it was only present in a minority of the sequences observed in the cerebrum of the cat USP16 (FIP+).

The transversions observed at position 10 of the $3 \mathrm{c}$ gene, which resulted in premature stop codons, were the same as those observed in the clone sequences obtained from the cerebrum and stomach of the FIP+ cats USP17 and USP6, respectively. With the exception of this finding and in agreement with the results of Pedersen et al. $[14,15]$, the $3 c$ gene mutations were unique to each cat, including the sequences obtained from the cohabitating FIP+ cats (USP17 and USP18).

It can be concluded that the presence of a truncated $3 c$ protein is a suggestive factor for differentiation between the FECV and FIPV pathotypes and therefore serves as a genetic marker of virulence. It still remains to be answered whether $3 \mathrm{c}$ is expendable for FCoV replication in phagocytic cells and a nontruncated $3 \mathrm{c}$ is thus selected-against during the FECV/FIPV transition. Moreover, the $3 \mathrm{a}-\mathrm{c}, \mathrm{E}$, and M genes showed a level of genetic diversity indicative of the constitution of FCoV quasispecies or mutant spectrum and the likelihood of emergence of a highly virulent, individualspecific biotype.

\section{Competing Interests}

None of the authors has any financial or personal relationships that could inappropriately influence or bias the content of the paper.

\section{Acknowledgments}

Funding for this study was provided by Fundação de Amparo à Pesquisa do Estado de São Paulo, Brazil (Grants nos. 09/17535-9 and 10/07492-8), Conselho Nacional de Desenvolvimento Científico e Tecnológico (Grant no. 300513/20100), and Coordenação de Aperfeiçoamento de Pessoal de Nível Superior (CAPES/PROEX 2327/2015). The authors are also grateful to the veterinarians and cat owners who provided FIP cases and cat faecal samples for this study.

\section{References}

[1] N. C. Pedersen, "A review of feline infectious peritonitis virus infection: 1963-2008," Journal of Feline Medicine and Surgery, vol. 11, no. 4, pp. 225-258, 2009.

[2] A. Kipar, M. L. Meli, K. E. Baptiste, L. J. Bowker, and H. Lutz, "Sites of feline coronavirus persistence in healthy cats," Journal of General Virology, vol. 91, no. 7, pp. 1698-1707, 2010.

[3] L. Vogel, M. van der Lubben, E. G. te Lintelo et al., "Pathogenic characteristics of persistent feline enteric coronavirus infection in cats," Veterinary Research, vol. 41, no. 5, article 71, 2010.
[4] P. S. Masters, “The molecular biology of coronaviruses," Advances in Virus Research, vol. 65, pp. 193-292, 2006.

[5] M. A. Brown, J. L. Troyer, J. Pecon-Slattery, M. E. Roelke, and S. J. O'Brien, "Genetics and pathogenesis of feline infectious peritonitis virus," Emerging Infectious Diseases, vol. 15, no. 9, pp. 1445-1452, 2009.

[6] C. Dye and S. G. Siddell, "Genomic RNA sequence of feline coronavirus strain FCoV C1Je," Journal of Feline Medicine and Surgery, vol. 9, no. 3, pp. 202-213, 2007.

[7] B. R. Bank-Wolf, I. Stallkamp, S. Wiese, A. Moritz, G. Tekes, and H.-J. Thiel, "Mutations of $3 \mathrm{c}$ and spike protein genes correlate with the occurrence of feline infectious peritonitis," Veterinary Microbiology, vol. 173, no. 3-4, pp. 177-188, 2014.

[8] H.-W. Chang, H. F. Egberink, R. Halpin, D. J. Spiro, and P. J. M. Rottie, "Spike protein fusion peptide and feline coronavirus virulence," Emerging Infectious Diseases, vol. 18, no. 7, pp. 10891095, 2012.

[9] C. S. Lewis, E. Porter, D. Matthews et al., "Genotyping coronaviruses associated with feline infectious peritonitis," Journal of General Virology, vol. 96, pp. 1358-1368, 2015.

[10] B. N. Licitra, J. K. Millet, A. D. Regan et al., "Mutation in spike protein cleavage site and pathogenesis of feline coronavirus," Emerging Infectious Diseases, vol. 19, no. 7, pp. 1066-1073, 2013.

[11] A. D. Regan, R. Shraybman, R. D. Cohen, and G. R. Whittaker, "Differential role for low $\mathrm{pH}$ and cathepsin-mediated cleavage of the viral spike protein during entry of serotype II feline coronaviruses," Veterinary Microbiology, vol. 132, no. 3-4, pp. 235-248, 2008.

[12] P. J. M. Rottier, K. Nakamura, P. Schellen, H. Volders, and B. J. Haijema, "Acquisition of macrophage tropism during the pathogenesis of feline infectious peritonitis is determined by mutations in the feline coronavirus spike protein," Journal of Virology, vol. 79, no. 22, pp. 14122-14130, 2005.

[13] L.-E. Hsieh, W.-P. Huang, D.-J. Tang, Y.-T. Wang, C.-T. Chen, and L.-L. Chueh, " $3 \mathrm{C}$ protein of feline coronavirus inhibits viral replication independently of the autophagy pathway," Research in Veterinary Science, vol. 95, no. 3, pp. 1241-1247, 2013.

[14] N. C. Pedersen, H. Liu, K. A. Dodd, and P. A. Pesavento, "Significance of coronavirus mutants in feces and diseased tissues of cats suffering from feline infectious peritonitis," Viruses, vol. 1, no. 2, pp. 166-184, 2009.

[15] N. C. Pedersen, H. Liu, J. Scarlett et al., "Feline infectious peritonitis: role of the feline coronavirus $3 c$ gene in intestinal tropism and pathogenicity based upon isolates from resident and adopted shelter cats," Virus Research, vol. 165, no. 1, pp. 1728, 2012.

[16] H. Vennema, A. Poland, J. Foley, and N. C. Pedersen, "Feline infectious peritonitis viruses arise by mutation from endemic feline enteric coronaviruses," Virology, vol. 243, no. 1, pp. 150157, 1998.

[17] A. Dedeurwaerder, D. A. J. Olyslaegers, L. M. B. Desmarets, I. D. M. Roukaerts, S. Theuns, and H. J. Nauwynck, "ORF7-encoded accessory protein $7 \mathrm{a}$ of feline infectious peritonitis virus as a counteragent against IFN- $\alpha$-induced antiviral response," Journal of General Virology, vol. 95, no. 2, pp. 393-402, 2014.

[18] M. Kennedy, N. Boedeker, P. Gibbs, and S. Kania, "Deletions in the $7 \mathrm{a}$ ORF of feline coronavirus associated with an epidemic of feline infectious peritonitis," Veterinary Microbiology, vol. 81, no. 3, pp. 227-234, 2001.

[19] C. M. Borschensky and M. Reinacher, "Mutations in the $3 \mathrm{c}$ and $7 \mathrm{~b}$ genes of feline coronavirus in spontaneously affected FIP 
cats," Research in Veterinary Science, vol. 97, no. 2, pp. 333-340, 2014.

[20] H.-W. Chang, R. J. de Groot, H. F. Egberink, and P. J. M. Rottier, "Feline infectious peritonitis: Insights into feline coronavirus pathobiogenesis and epidemiology based on genetic analysis of the viral 3c gene," Journal of General Virology, vol. 91, no. 2, pp. 415-420, 2010.

[21] A. S. Hora, K. M. Asano, J. M. Guerra et al., "Intrahost diversity of feline coronavirus: a consensus between the circulating virulent/avirulent strains and the internal mutation hypotheses?" The Scientific World Journal, vol. 2013, Article ID 572325, 8 pages, 2013.

[22] T. A. Hall, "Bioedit: a user-friendly biological sequence alignment editor and analysis program for Windows 95/98/NT," in Nucleic Acids Symposium Series, vol. 41, pp. 95-98, 1999.

[23] K. Tamura, D. Peterson, N. Peterson, G. Stecher, M. Nei, and S. Kumar, "MEGA5: molecular evolutionary genetics analysis using maximum likelihood, evolutionary distance, and maximum parsimony methods," Molecular Biology and Evolution, vol. 28, no. 10, pp. 2731-2739, 2011.

[24] Á. Bálint, A. Farsang, Z. Zádori, and S. Belák, "Comparative in vivo analysis of recombinant type II feline coronaviruses with truncated and completed ORF3 region," PLoS ONE, vol. 9, no. 2, Article ID e88758, 2014.

[25] P. J. M. Rottier, “The coronavirus membrane glycoprotein," in The Coronaviridae, S. G. Siddell, Ed., pp. 115-140, Plenum Press, New York, NY, USA, 1995. 

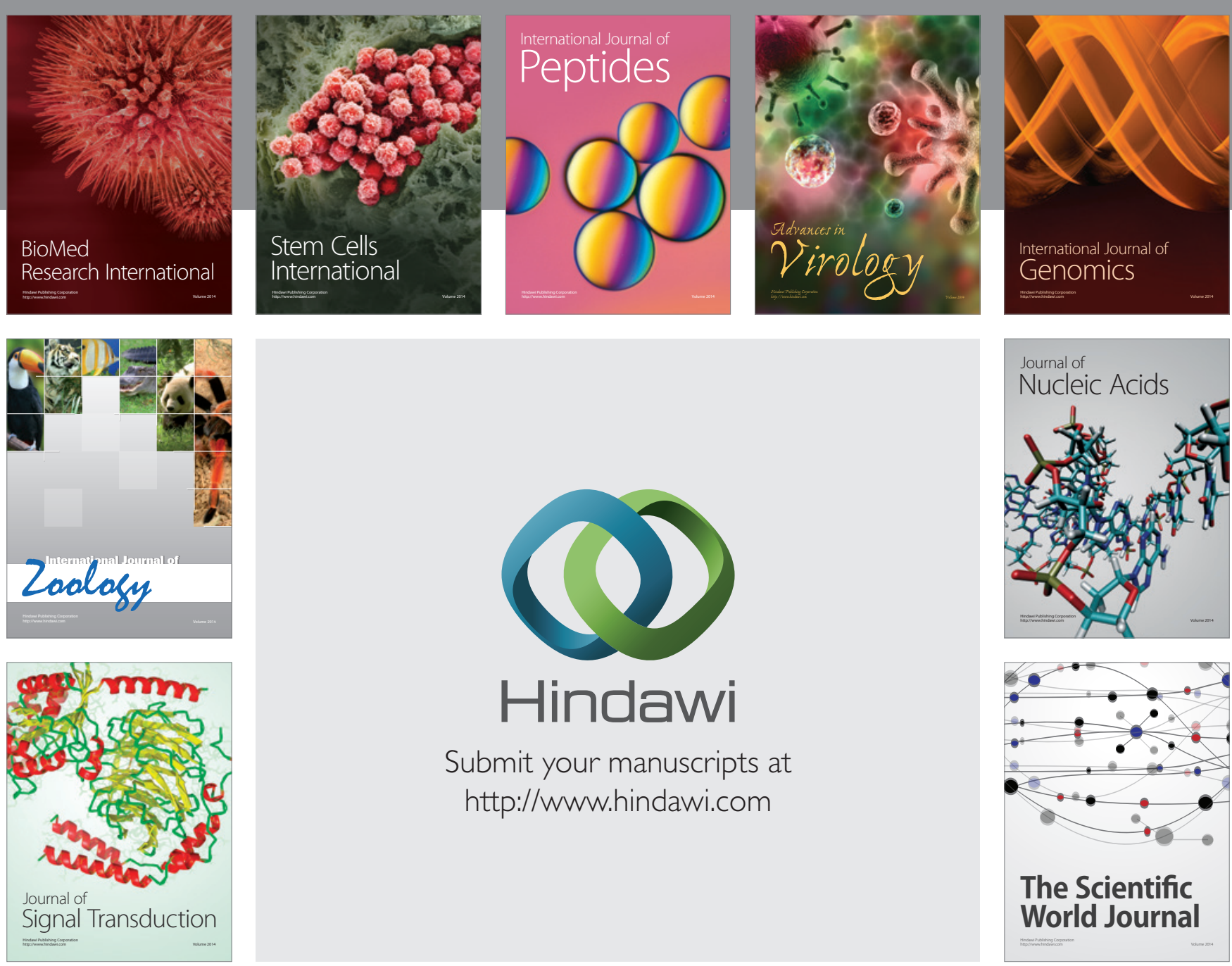

Submit your manuscripts at

http://www.hindawi.com
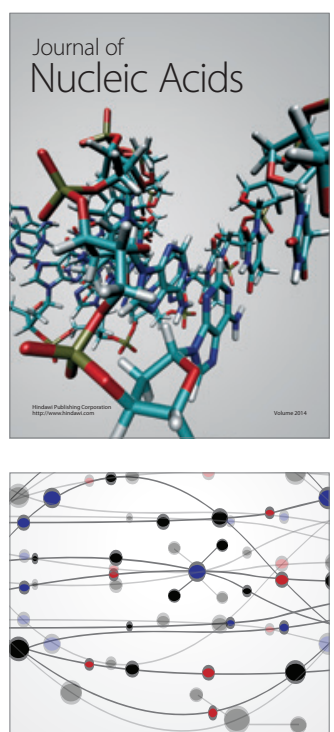

The Scientific World Journal
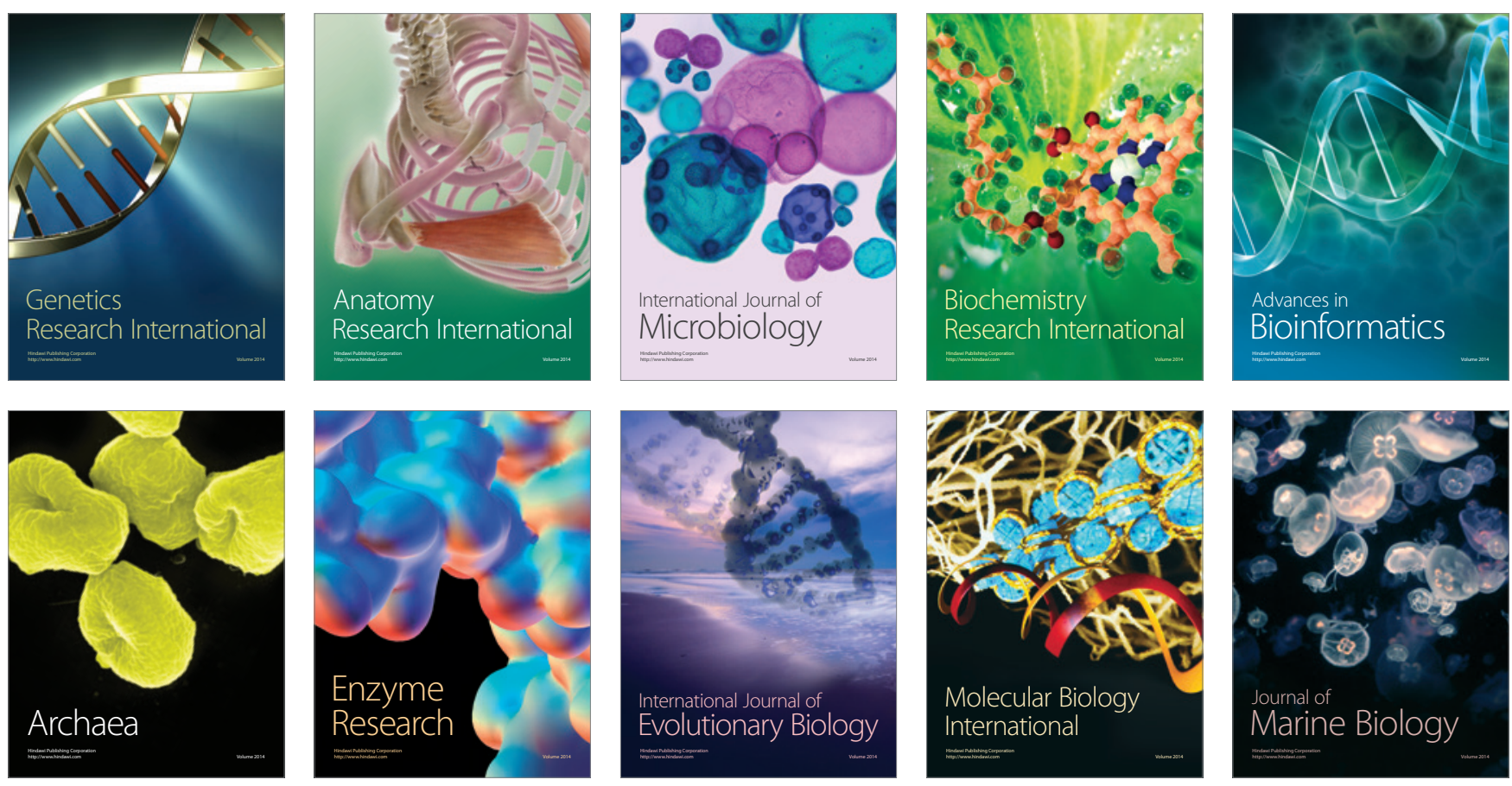